\title{
Desenvolvimento e reprodução da mosca-do-mediterrâneo em caquizeiro, macieira, pessegueiro e videira
}

\author{
Odimar Zanuzo Zanardi(1), Dori Edson Nava(2), Marcos Botton ${ }^{(3)}$, Anderson Dionei Grützmacher ${ }^{(1)}$, \\ Ruben Machota Jr(1) e Maicon Bisognin ${ }^{(1)}$
}

\begin{abstract}
(1)Universidade Federal de Pelotas, Faculdade de Agronomia Eliseu Maciel, Departamento de Fitossanidade, Campus Universitário, s/no, Caixa Postal 354, CEP 96010-900 Pelotas, RS. E-mail: odimarzanardi@yahoo.com.br, anderson.grutzmacher@pq.cnpq.br, ruben_soad@yahoo.com.br, maicon.bisognin@yahoo.com.br (2)Embrapa Clima Temperado, Rodovia BR 392, Km 78, Caixa Postal 403, CEP 96010-971 Pelotas, RS. E-mail: nava.dori@cpact.embrapa.br ${ }^{(3)}$ Embrapa Uva e Vinho, Rua Livramento № 515, Caixa Postal 130, CEP 95700-000 Bento Gonçalves, RS. E-mail: marcos@cnpuv.embrapa.br
\end{abstract}

Resumo - O objetivo deste trabalho foi descrever o desenvolvimento e reprodução de Ceratitis capitata (Diptera: Tephritidae) em frutos de caqui 'Fuyu', maçã 'Gala', pêssego 'Maciel' e uva 'Itália'. Foram feitas avaliações quanto a: duração e viabilidade do ovo, duração do estágio larval, peso de pupa, duração e viabilidade da pupa, duração do período ovo-adulto, razão sexual, período de pré-oviposição e oviposição, fecundidade diária e total e longevidade de machos e fêmeas. A maior duração do período ovo-adulto ocorreu em maçã, em consequência do aumento do estágio larval, seguido por uva, caqui e pêssego. Larvas que se alimentaram de pêssego apresentaram maior peso médio de pupa. A maior viabilidade pupal foi registrada em pêssego, equivalente à de uva e caqui. O período de pré-oviposição foi maior para fêmeas provenientes de maçã e uva. Fêmeas provenientes de pêssego apresentaram período de oviposição mais longo, além de maior fecundidade média diária e fecundidade total. A longevidade de adultos foi superior em insetos criados em pêssego. Frutos de pessegueiro são mais adequados para o desenvolvimento de C. capitata, em comparação aos de caqui, maçã e uva.

Termos para indexação: Ceratitis capitata, desenvolvimento larval, fruticultura temperada, mosca-das-frutas, reprodução.

\section{Development and reproduction of the Mediterranean fruit fly in persimmon, apple, peach and grape}

\begin{abstract}
The objective of this work was to describe the development and reproduction of Ceratitis capitata (Diptera: Tephritidae) in fruit of 'Fuyu' persimmon, 'Gala' apple, 'Maciel' peach and 'Italy' grape. Evaluations were made for: egg duration and viability, duration of larval stage, pupa weight, pupal duration and viability, duration of egg-adult period, sex ratio, pre-oviposition and oviposition periods, daily and total fecundity, and longevity of males and females. The longer duration of the egg-adult period occurred in apple, due to the increase in larval stage, followed by grape, persimmon and peach. Larvae fed on peach had higher average pupal weight. The highest pupal viability was recorded in peach and was equivalent to that of grape and persimmon. The pre-oviposition period was higher for females from apple and grape. Females from peach had longer oviposition period, and higher daily average fecundity and total fertility. The longevity of adults was higher in insects reared on peach. Peach fruit are more suitable for the development of C. capitata, in comparison to persimmon, apple and grape.
\end{abstract}

Index terms: Ceratitis capitata, larval development, temperate fruit crop, fruit fly, reproduction.

\section{Introdução}

O Estado do Rio Grande do Sul é o principal produtor de frutas de clima temperado, com destaque para o caquizeiro (Diospyros kaki L.), macieira (Malus domestica Borkhausen, 1803), pessegueiro [Prunus persica (L.) Batsch., 1801] e videira (Vitis spp.), com total de produção de 1,4 milhões de toneladas de frutas em 81.507 ha (Instituto Brasileiro de Geografia e Estatística, 2011).

Um dos fatores limitantes ao aumento da produtividade das frutíferas é a ocorrência de insetos-praga, entre os quais a mosca-das-frutas sul-americana Anastrepha fraterculus Wiedemann, 1830 (Diptera: Tephritidae) é a mais frequente e abundante nos pomares (Scoz et al., 2004; 
Zart et al., 2010). No entanto, nos últimos anos, tem sido observada uma mudança na ocorrência dos tefritídeos, principalmente na metade sul do Estado, com aumento significativo na população da mosca-do-mediterrâneo, Ceratitis capitata Wiedemann, 1824 (Diptera: Tephritidae), próximo aos pomares comerciais (Nava et al., 2008). A mosca-do-mediterrâneo caracteriza-se por ser uma espécie polífaga, cosmopolita, multivoltina e com alto potencial para causar danos (Joachim-Bravo et al., 2001b), além de dispor de ampla variedade de hospedeiros nativos e cultivados e ter alta capacidade de adaptação a novos nichos ecológicos (Copeland et al., 2002; Raga et al., 2005). Entre as espécies hospedeiras de $C$. capitata importantes para a fruticultura de clima temperado, na Região Sul do Brasil, destacamse o caquizeiro, a macieira, o pessegueiro e a videira (Zucchi, 2001).

Os danos causados pela mosca-do-mediterrâneo são decorrentes da introdução do ovipositor das fêmeas na epiderme dos frutos e do desenvolvimento das larvas no seu interior. Além dos danos diretos, a infestação de $C$. capitata restringe a exportação de frutas frescas, em razão das barreiras quarentenárias impostas pelos países importadores (Souza-Filho et al., 2003).

Embora seja uma das espécies mais estudadas em outras regiões do Brasil e do mundo, pouco se conhece, no Sul do Brasil, sobre a biologia de $C$. capitata em frutíferas de clima temperado. A recente expansão da fruticultura no sul do Estado, aliada ao grande número de hospedeiros de C. capitata disponíveis, torna necessário investigar o potencial biológico da espécie nas principais frutíferas de clima temperado cultivadas na região.

O objetivo deste trabalho foi descrever o desenvolvimento e a reprodução de $C$. capitata, em frutos de caquizeiro, macieira, pessegueiro e videira.

\section{Material e Métodos}

Para o estudo da biologia de C. capitata foram utilizados 75 frutos maduros (maturação comercial) de caqui 'Fuyu', maçã 'Gala', pêseego 'Maciel', além de 120 bagas de uva 'Itália', provenientes de pomares sem aplicação de inseticidas por, pelo menos, 30 dias antes da coleta dos frutos. Antes da instalação do experimento, colheram-se três amostras de dez frutos, que foram analisadas quanto aos teores de sólidos solúveis totais (SST), acidez titulável (AT) e pH, para a caracterização química.
O experimento foi conduzido em sala climatizada (temperatura de $25 \pm 2^{\circ} \mathrm{C}$, umidade relativa do ar de $75 \pm 15 \%$ e fotofase de 12 horas), no Laboratório de Entomologia da Embrapa Clima Temperado, com insetos adultos provenientes da criação de manutenção de C. capitata, onde se utilizam frutos de mamão papaia (Carica papaya Linnaeus, 1753) como substrato de oviposição e desenvolvimento larval. Os insetos que originaram essa criação foram coletados em frutos de pessegueiro, caquizeiro e araçazeiro (Psidium cattleyanum Sabine, 1821), no Município de Pelotas, RS, em 2009.

Os adultos foram mantidos em gaiolas de madeira $(50 \times 45 \times 40 \mathrm{~cm})$, revestidas com tecido voile, com uma abertura lateral, para a introdução dos insetos e dos frutos. Nessas gaiolas, os adultos foram alimentados com dieta sólida de proteína de soja, gérmen de trigo e levedura de cerveja, na proporção de 3:1:1, suplementada com uma mistura de levedura de cerveja e mel na proporção de 2:1, respectivamente. As dietas foram oferecidas em placas de Petri $(4,5 \mathrm{~cm}$ de diâmetro) e a água em um recipiente de plástico com algodão hidrófilo embebido em água destilada.

Para cada espécie de fruto, foram utilizadas cinco gaiolas, com 15 frutos e 72 bagas de uva por gaiola, com insetos de sete a oito dias de idade após a emergência, em delineamento inteiramente casualizado. Em cada gaiola, foi mantida a proporção de três casais por fruto, no total de 225 casais para os tratamentos com caqui, maçã e pêssego e 360 casais para uva. Os frutos foram expostos às moscas para oviposição pelo período de 24 horas.

Após esse período, os frutos foram retirados das gaiolas e colocados individualmente em recipientes de plástico de $750 \mathrm{~mL}$, tampados com tecido tipo voile fixado com atilho de borracha, para permitir aeração e evitar a fuga das larvas. No fundo de cada recipiente, foi colocada uma camada de $2 \mathrm{~cm}$ de vermiculita fina, para absorver o excesso de umidade.

Para a determinação do período de incubação dos ovos, foi realizado um experimento paralelo, com dez frutos de cada hospedeiro, tendo-se utilizado a mesma metodologia. Os frutos foram avaliados a cada 12 horas, com remoção da epiderme para observação da presença de larvas eclodidas, com auxílio de microscópio estereoscópico binocular com aumento de 40x.

A duração do estágio larval foi determinada por meio da inspeção diária da vermiculita das unidades 
experimentais (recipientes com frutos), do terceiro dia após a oviposição ao final da constatação da presença de pupários na vermiculita. Os pupários obtidos de cada fruto foram individualizados e colocados em recipientes de acrílico $(25 \mathrm{~mL})$, que continham vermiculita de textura fina umedecida, até a emergência dos adultos para determinação da duração do estágio pupal. Os pupários foram pesados com uma balança analítica, com precisão de 0,0001 g, modelo E02140, (Ohaus Corporation, Toledo Brasil, São Bernardo do Campo, SP, Brasil), 24 horas após a retirada do recipiente com o fruto (Zart et al., 2010).

A viabilidade pupal foi determinada pela relação entre o número de adultos emergidos e o número de pupários obtidos em cada fruto. A razão sexual (rs) dos adultos emergidos foi determinada pela equação rs = fêmea/(fêmea + macho), proposta por Silveira Neto et al. (1976). Após a emergência, separaramse os adultos por sexo e, em seguida, formaram-se casais para a avaliação dos seguintes parâmetros da fase adulta: período de pré-oviposição e oviposição, fecundidade diária e total e longevidade de machos e fêmeas. Os casais foram colocados no interior de gaiolas, confeccionadas com recipientes de plástico transparente de $500 \mathrm{~mL}$, cuja abertura foi apoiada sobre uma placa de acrílico (Germbox), com o fundo perfurado com orifícios de $2 \mathrm{~mm}$ de diâmetro, para a circulação de ar. Em cada gaiola, foi disponibilizada a dieta, fornecida em recipientes de plástico com capacidade de 4,0 g, e água em recipientes de acrílico com capacidade para $10 \mathrm{~mL}$, por meio de rolete dental de algodão hidrófilo embebido em água destilada.

Os ovos foram obtidos por meio de "fruto artificial", constituído de 75,0 $\mathrm{mL}$ de suco de amora, 4,0 $\mathrm{g}$ de ágar, $0,4 \mathrm{~g}$ de metilparahidroxibenzoato (Nipagin) e $350,0 \mathrm{~mL}$ de água destilada, envoltos com parafilme, conforme Salles (1992). Os "frutos artificiais" foram expostos a casais de $C$. Capitata e substituídos a cada 24 horas, para a determinação da fecundidade diária e total.

Para avaliar o efeito do hospedeiro, utilizado como alimento da fase larval, sobre a duração e viabilidade do estágio de ovo, foram utilizados 20 ovos da segunda e terceira oviposição de cada casal. Os ovos foram retirados dos "frutos artificiais", com auxílio de pincel fino, e colocados sobre tecido de algodão umedecido, no interior de placas de Petri, envoltas com filme de PVC e mantidas em câmara climatizada BOD (Papadopoulos et al., 2002). Diariamente, as larvas eclodidas foram contadas e retiradas para determinação da duração e viabilidade da fase de ovo.

Os dados de cada variável biológica foram analisados quanto à homocedasticidade, transformados em $1 / \mathrm{x}^{1 / 2}$ (longevidade de machos e fêmeas), e analisados pelo teste F com o SAS (SAS Institute, 2002). As médias foram comparadas pelo teste de Tukey, a 5\% de probabilidade.

\section{Resultados e Discussão}

O período de incubação dos ovos foi de aproximadamente dois dias, independentemente do tipo de fruto utilizado para o desenvolvimento larval (Tabela 1). Esse valor é próximo ao reportado por Papachristos et al. (2008), que observaram que o período de incubação de ovos de C. capitata é de dois a três dias, a $25^{\circ} \mathrm{C}$. Papadopoulos et al. (2002) avaliaram os parâmetros demográficos da mosca-do-mediterrâneo em maçãs 'Golden Delicious' e constataram que a maioria das larvas eclodiu nas primeiras 48 horas após a oviposição.

A viabilidade de ovos não foi afetada pelo alimento utilizado na fase larval, e ficou em torno de $70 \%$, nos quatro hospedeiros analisados, embora Papadopoulos et al. (2002) tenham obtidos valores de viabilidade de 93,5\% em maçãs 'Golden Delicious'.

A duração do estágio larval diferiu significativamente entre os hospedeiros e foi maior em maçã 'Gala', seguida pela uva 'Itália', caqui 'Fuyu' e pêssego 'Maciel' (Tabela 1). Papadopoulos et al. (2002) verificaram que a duração média do estágio larval de C. capitata, em maçã 'Golden Delicious', foi de 18 dias sob temperatura de $25 \pm 2^{\circ} \mathrm{C}$. No entanto, Gómez et al. (2008) relataram que o período ovopupa foi de 19 dias em duas cultivares de uva de mesa, Benitaka e Festival. Thomas et al. (2010) verificaram que, em pêssego, esse período varia de 10 a 15 dias, semelhantemente ao observado no presente trabalho. O menor período de desenvolvimento obtido em pêssego indica que esta fruta é a mais adequada para o desenvolvimento do inseto, em comparação ao caqui, maçã e uva. Joachim-Bravo et al. (2001a), ao avaliar o desenvolvimento de larvas de $C$. capitata em frutos de maçã e mamão, concluíram que maçã não é um hospedeiro adequado para o desenvolvimento do inseto.

De acordo com Cresoni-Pereira \& Zucoloto (2009), larvas de $C$. capitata necessitam de carboidratos, 
proteínas, lipídeos, sais minerais e vitaminas para um desenvolvimento adequado. Kaspi et al. (2002) constataram que, quanto maior o nível de proteína presente no alimento ingerido na fase imatura, menor é o tempo de desenvolvimento do inseto. Os componentes nutricionais e energéticos básicos, presentes em $100 \mathrm{~g}$ de frutos de caqui, maçã, pêssego e uva, são em média de: $0,61,0,20,0,80$ e $1,00 \mathrm{~g}$ de proteínas; 0,33, 0,36, 0,16 e 15,25 g de lipídeos totais; e 17,20, 15,25, 13,30 e $9,30 \mathrm{~g}$ de carboidratos totais, respectivamente (Valderrama et al., 2001; Elias et al., 2008).

O tempo de desenvolvimento larval de C. capitata, em maçã, apresentou maior amplitude em comparação a caqui, pêssego e uva. O período para obtenção do primeiro e do último pupário, em maçã, foi de 14 dias, enquanto nos demais hospedeiros esse intervalo foi de cinco a oito dias. Esses resultados são semelhantes aos reportados por Papadopoulos et al. (2002), que observaram que, em maçã, aproximadamente $60 \%$ das larvas deixaram os frutos, para se transformar em pupa do $13^{\circ}$ ao $17^{\circ}$ dias, enquanto $10 \%$ se transformaram no $25^{\circ}$ e $27^{\circ}$ dias após a oviposição.

O maior peso médio de pupa foi observado em pêssego seguido por caqui, uva e maçã, e diferiu significativamente entre os hospedeiros (Tabela 1). De acordo com Plácido-Silva et al. (2005), alimentos com baixo teor de proteínas, como é o caso de frutos de maçã, reduzem o tamanho das larvas e, consequentemente, o peso do pupário. Embora a uva apresente maior valor de proteína total, o tamanho do fruto é menor, e pode haver competição das larvas pelo alimento. Além disso, a uva apresenta maior quantidade de ácidos solúveis e menor valor de $\mathrm{pH}$, o que pode afetar o desenvolvimento do inseto no interior da baga. De acordo com Papachristos et al. (2008), o pH ideal para o desenvolvimento de $C$. capitata situa-se em torno de 4,0. No entanto, as bagas de uva utilizadas no estudo apresentavam valores de $\mathrm{pH}$ de 2,56 , o que pode ser um dos fatores responsáveis pelo atraso no desenvolvimento do inseto.

A duração do estágio pupal foi semelhante entre os hospedeiros analisados e variou de 9,2 a 11,0 dias (Tabela 1). Esses resultados se assemelham aos obtidos por Papadopoulos et al. (2002), que verificaram que a duração do estágio pupal foi de dez dias para C. capitata em maçã 'Golden Delicious'. Medeiros et al. (2007) também observaram que a duração do estágio pupal, em sete hospedeiros, variou de 9,3 a 11,9 dias. De acordo com Thomas et al. (2010), a duração do estágio pupal de $C$. capitata pode variar de 6 a 13 dias conforme o hospedeiro e as condições climáticas do local.

Os insetos que utilizaram pêssegos como alimento, durante o estágio larval, apresentaram maior viabilidade de pupa, equivalente à dos alimentados com uva e caqui, porém diferiram significativamente daqueles provenientes de maçã (Tabela 1). Gómez et al. (2008) avaliaram o desenvolvimento de $C$. capitata ${ }_{2}$ em uva de mesa, e constataram que a viabilidade pupal foi de 55 e $80 \%$, para insetos que utilizaram uvas 'Festival' e 'Benitaka' como alimento durante o estágio larval, respectivamente. No entanto, Joachim-Bravo et al. (2001b) registraram emergência de apenas 16,7\%

Tabela 1. Variáveis biológicas de Ceratitis capitata, criada em frutos de caquizeiro, macieira, pessegueiro e videira em laboratório ${ }^{(1)}$.

\begin{tabular}{|c|c|c|c|c|c|}
\hline Variável biológica & Caquizeiro & Macieira & Pessegueiro & Videira & $\mathrm{CV}(\%)$ \\
\hline Duração do estágio de ovo (dias) & $2,07 \pm 0,16 \mathrm{a}$ & $2,19 \pm 0,17 \mathrm{a}$ & $2,11 \pm 0,15 \mathrm{a}$ & $2,38 \pm 0,19 \mathrm{a}$ & 24,68 \\
\hline Viabilidade de ovo (\%) & $70,93 \pm 2,55 \mathrm{a}$ & $69,45 \pm 2,43 a$ & $72,78 \pm 2,55 \mathrm{a}$ & $70,38 \pm 2,13 a$ & 17,74 \\
\hline Duração do estágio larval (dias) & $16,97 \pm 0,18 \mathrm{c}$ & $21,07 \pm 0,16 \mathrm{a}$ & $11,34 \pm 0,19 \mathrm{~d}$ & $18,20 \pm 0,19 b$ & 12,51 \\
\hline Peso de pupas (mg) & $8,94 \pm 0,10 \mathrm{~b}$ & $7,98 \pm 0,10 \mathrm{~d}$ & $9,80 \pm 0,11 \mathrm{a}$ & $8,47 \pm 0,16 \mathrm{c}$ & 15,56 \\
\hline Duração do estágio pupal (dias) & $9,22 \pm 1,02 \mathrm{a}$ & $10,78 \pm 1,02 \mathrm{a}$ & $10,11 \pm 0,65 \mathrm{a}$ & $11,00 \pm 0,58 \mathrm{a}$ & 17,22 \\
\hline Viabilidade de pupa (\%) & $80,76 \pm 2,77 \mathrm{ab}$ & $70,78 \pm 3,63 b$ & $85,45 \pm 3,37 \mathrm{a}$ & $82,30 \pm 3,86 a b$ & 23,17 \\
\hline Duração do período ovo-adulto (dias) & $29,36 \pm 0,43 \mathrm{c}$ & $35,14 \pm 0,53 \mathrm{a}$ & $24,77 \pm 0,36 \mathrm{~d}$ & $32,55 \pm 0,68 \mathrm{~b}$ & 11,35 \\
\hline Razão sexual & $0,47 \pm 0,02 \mathrm{a}$ & $0,49 \pm 0,02 \mathrm{a}$ & $0,49 \pm 0,01 \mathrm{a}$ & $0,48 \pm 0,03 \mathrm{a}$ & 7,80 \\
\hline Período de pré-oviposição (dias) & $4,90 \pm 0,43 b$ & $11,74 \pm 0,89 \mathrm{a}$ & $5,30 \pm 0,12 b$ & $10,43 \pm 0,49 \mathrm{a}$ & 31,08 \\
\hline Período de oviposição (dias) & $31,70 \pm 2,70 \mathrm{~b}$ & $33,00 \pm 2,88 \mathrm{~b}$ & $43,35 \pm 4,28 \mathrm{a}$ & $33,13 \pm 4,71 b$ & 49,52 \\
\hline Fecundidade diária & $11,52 \pm 1,06 \mathrm{a}$ & $6,03 \pm 0,52 b$ & $11,91 \pm 0,88 \mathrm{a}$ & $8,17 \pm 1,13 b$ & 47,30 \\
\hline Fecundidade total & $363,87 \pm 49,40 \mathrm{a}$ & $192,22 \pm 20,70 b$ & $434,48 \pm 40,47 \mathrm{a}$ & $206,78 \pm 31,52 b$ & 44,83 \\
\hline Longevidade de macho (dias) & $49,72 \pm 4,61 \mathrm{~b}$ & $44,16 \pm 3,72 b$ & $79,16 \pm 3,67 \mathrm{a}$ & $52,60 \pm 4,86 \mathrm{~b}$ & 38,28 \\
\hline Longevidade da fêmea (dias) & $46,68 \pm 3,31 \mathrm{~b}$ & $47,12 \pm 3,15 b$ & $63,56 \pm 5,12 \mathrm{a}$ & $42,44 \pm 5,04 \mathrm{~b}$ & 43,18 \\
\hline
\end{tabular}

${ }^{(1)}$ Médias \pm amplitude de variação seguidas de letras iguais, nas linhas, não diferem entre si pelo teste de Tukey, a $5 \%$ de probabilidade. CV, coeficiente de variação. 
em maçã (Pyrus malus L.). Ovruski et al. (2010), ao avaliar a ocorrência de $C$. capitata em hospedeiros exóticos no Vale do Tucumã, Noroeste da Argentina, relataram que a viabilidade pupal variou de 8 a $22 \%$, para insetos que utilizaram maçã como hospedeiro, e $35 \%$ em pessegueiros, enquanto Zucoloto (1993) registrou apenas 54\% de emergência. No entanto, Medeiros et al. (2007) verificaram que a viabilidade pupal foi superior a $93 \%$, em pessegueiro.

As diferenças observadas na emergência estão relacionadas às variações nas populações de C. capitata, características nutricionais e físico-químicas dos hospedeiros, além da presença de fatores antinutricionais nos frutos, como taninos, antocianinas, carotenoides, ácidos e aleloquímicos (Zucoloto, 1993; Plácido-Silva et al., 2005).

A duração do período ovo-adulto diferiu entre os quatro hospedeiros estudados, e o maior valor foi observado em insetos que utilizaram maçã como alimento durante a fase imatura (Tabela 1). Em maçã, Papadopoulos et al. (2002) observaram que o hospedeiro proporcionou aumento no ciclo biológico do inseto, que totalizou 53 dias. Em uva, Gómez et al. (2008) verificaram que o período ovo-adulto de $C$. capitata foi de 30 dias, à temperatura de $25^{\circ} \mathrm{C}$, semelhante ao obtido neste trabalho. Souza-Filho et al. (2003) relataram que o período ovo-adulto de $C$. capitata varia de 17 a 26 dias, a depender do hospedeiro e das condições climáticas. De acordo com Plácido-Silva et al. (2005), o alimento ingerido na fase larval influencia diretamente o ciclo biológico do inseto, o que pode resultar em variações no número de gerações anuais e na densidade populacional da praga.

A razão sexual não apresentou diferenças significativas entre os hospedeiros estudados e variou de 0,47 a 0,49. Esses resultados são semelhantes aos obtidos por Medeiros et al. (2007), que avaliaram a razão sexual de $C$. capitata em sete hospedeiros e também verificaram relação de aproximadamente um macho para cada fêmea.

O maior período de pré-oviposição foi observado em insetos provenientes de uva e maçã, e diferiu significativamente daqueles oriundos de caqui e pêssego (Tabela 1). Papadopoulos et al. (2002) registraram que fêmeas provenientes de maçã apresentaram período de pré-oviposição de 12,5 dias. Fernandes-da-Silva \& Zucoloto (1997) estudaram o efeito do hospedeiro sobre ovos de $C$. capitata e verificaram que os insetos que utilizaram alimentos mais nutritivos (proteicos e energéticos) apresentaram período de pré-oviposição relativamente curto, de apenas cinco dias.

Fêmeas provenientes de pêssegos apresentaram maior período de oviposição (Tabela 1). Atribui-se esse resultado às diferenças qualitativas, proporcionadas pelo hospedeiro, que garantiram ao inseto maior desenvolvimento das estruturas reprodutivas. No entanto, Papadopoulos et al. (2002) observaram que larvas criadas em maçã 'Golden Delicious' apresentaram período de oviposição de 32,3 dias, similar ao reportado no presente trabalho para fêmeas provenientes de maçã 'Gala'.

Os maiores valores para fecundidade média diária e total foram obtidos em fêmeas provenientes de frutos de pêssego e caqui, que diferiram significativamente de uva e maçã (Tabela 1). Papadopoulos et al. (2002) observaram que, em fêmeas provenientes de maçã, as fecundidades médias, diária e total foram de 11 e 555,1 ovos, respectivamente, valores superiores aos obtidos no presente trabalho para maçã 'Gala'. Valores de fecundidade total de 200 e 153 ovos por fêmea foram reportados por Gómez et al. (2008), em insetos criados em uva 'Benitaka' e 'Festival', respectivamente. Esses resultados indicam que o alimento utilizado durante o desenvolvimento da fase imatura afeta significativamente o desempenho reprodutivo dos insetos na fase adulta (Joachim-Bravo et al., 2010), o que pode influenciar a dinâmica populacional do inseto.

A maior longevidade de adultos foi observada em insetos provenientes de pêssego, e diferiu dos demais tratamentos (Tabela 1). Estudo realizado por Papadopoulos et al. (2002), com maçã 'Golden Delicious', indicou que a sobrevivência de adultos foi superior a $80 \%$ até 40 dias, seguido de uma alta mortalidade entre 40 e 70 dias após a emergência, semelhantemente aos resultados obtidos no presente trabalho.

Com base nos resultados obtidos, observou-se que o pessegueiro foi o hospedeiro que proporcionou o melhor desenvolvimento de $C$. capitata, seguido do caquizeiro, de videira e da macieira. Estudos realizados em diferentes regiões produtoras do mundo têm verificado altas infestações de $C$. capitata em pessegueiro (Zeki et al., 2008; Souza-Filho et al., 2009). No entanto, o caqui 'Fuyu', a maçã 'Gala' e a uva 'Itália', embora sejam menos adequados ao inseto, permitem 
o desenvolvimento e a multiplicação da espécie. No Rio Grande do Sul, onde os pomares de fruteiras de clima temperado geralmente são diversificados e localizados em pequenas propriedades, próximas entre si, a mosca-do-mediterrâneo pode infestar primeiramente frutos de pessegueiro, a partir de outubro, migrar para a videira (colheita de janeiro a março), e passar para pomares de macieiras e caquizeiros, cuja colheita é mais tardia (fevereiro a maio). Ao se considerar a elevada capacidade de dispersão e o alto potencial biótico da praga (Lopes et al., 2006), todas as frutíferas de clima temperado estudadas são suscetíveis aos danos causados pelo inseto; portanto, é recomendável que se adotem medidas de manejo.

\section{Conclusões}

1. Pêssego 'Maciel', caqui 'Fuyu', uva 'Itália' e maçã 'Gala' permitem o desenvolvimento biológico de Ceratites capitata.

2. Pêssego 'Maciel' é o hospedeiro que propicia o melhor desenvolvimento biológico de C. capitata, em comparação a caqui 'Fuyu', uva 'Itália' e maçã 'Gala'.

\section{Agradecimento}

Ao Conselho Nacional de Desenvolvimento Científico e Tecnológico, pelo apoio financeiro.

\section{Referências}

COPELAND, R.S.; WHARTON, R.A.; LUKE, Q.; MEYER, M. Indigenous hosts of Ceratitis capitata (Diptera: Tephritidae) in Kenya. Annals of the Entomological Society of America, v.95, p.672-694, 2002.

CRESONI-PEREIRA, C.; ZUCOLOTO, F.S. Moscas-das-frutas (Diptera). In: PANIZZI, A.R.; PARRA, J.R.P. (Ed.). Bioecologia e nutrição de insetos: base para o manejo integrado de pragas. Brasília: Embrapa Informação Tecnológica; Londrina: Embrapa Soja, 2009. p.733-768.

ELIAS, N. de F.; BERBERT, P.A.; MOLINA, M.A.B. de; VIANA, A.P.; DIONELLO, R.G.; QUEIROZ, V.A.V. Avaliação nutricional e sensorial de caqui cv. Fuyu submetido à desidratação osmótica e secagem por convecção. Ciência e Tecnologia de Alimentos, v.28, p.322-328, 2008.

FERNANDES-DA-SILVA, P.G.; ZUCOLOTO, F.S. Effect of host nutritive value on egg production by Ceratitis capitata (Diptera: Tephritidae). Journal of Insect Physiology, v.43, p.939-943, 1997.
GÓMEZ, M.; PARANHOS, B.J.; DAMASCENO, I.; CASTRO, R.; CAMPO, D.; ANDRADE, K.; SILVA, M.; NASCIMENTO, A.S.; MALAVASI, Y.A. Biología de la mosca del mediterráneo, Ceratitis capitata Wiedemann (Diptera: Tephritidae) en dos variedades de uva de mesa (Vitis vinifera L.) en el Nordeste brasileño. CitriFrut, v.25, p.18-23, 2008.

INSTITUTO BRASILEIRO DE GEOGRAFIA E ESTATÍSTICA. Sistema IBGE de Recuperação Automática (SIDRA). 2009. Disponível em: <http:/www.sidra.ibge.gov.br/bda/agric/default.a $\mathrm{sp} ? \mathrm{t}=4 \& \mathrm{z}=\mathrm{t} \& \mathrm{o}=11 \& \mathrm{u} 1=1 \& \mathrm{u} 2=34 \& \mathrm{u} 3=1 \& \mathrm{u} 5=1 \& \mathrm{u} 6=1 \& \mathrm{u} 4=34>$. Acesso em: 10 abr. 2011.

JOACHIM-BRAVO, I.S.; FERNANDES, O.A.; BORTOLI, S.A. de; ZUCOLOTO, F.S. Oviposition behavior of Ceratitis capitata Wiedemann (Diptera: Tephritidae): association between oviposition preference and larval performance in individual females. Neotropical Entomology, v.30, p.559-564, 2001a.

JOACHIM-BRAVO, I.S.; FERNANDES, O.A.; BORTOLI, S.A. de; ZUCOLOTO, F.S. Oviposition preference hierarchy in Ceratitis capitata (Diptera, Tephritidae): influence of female age and experience. Iheringia. Série Zoologia, v.91, p.93-100, 2001 b.

JOACHIM-BRAVO, I.S.; GUIMARAES, A.N.; MAGALHAES, T.C.; NASCIMENTO, A.S. Ecology, behavior and bionomics: performance de Ceratitis capitata Wiedemann (Diptera: Tephritidae) em frutos: comparação de duas populações criadas em laboratório. Neotropical Entomology, v.39, p.9-14, 2010.

KASPI, R.; MOSSINSON, S.; DREZNER, T.; KAMENSKY, B.; YUVAL, B. Effects of larval diet on development rates and reproductive maturation of male and female Mediterranean fruit flies. Physiological Entomology, v.27, p.29-38, 2002.

LOPES, D.J.H.; PIMENTEL, R.; NUNES, L.V.; COSTA, R.; SILVA, M.L.O.; SILVA, D.; MUMFORD, J.; MEXIA, A. Contributo do projecto interfruta para o conhecimento da dispersão da mosca do mediterrâneo (Ceratitis capitata Wiedmann) (Diptera: Tephritidae) na Ilha Terceira, Açores. Boletín de Sanidad Vegetal. Plagas, v.32, p.391-397, 2006.

MEDEIROS, A.; OLIVEIRA, L.; GARCIA, P. Suitability as Medfly Ceratitis capitata (Diptera, Tephritidae) hosts, of seven fruit species growing on the Island of São Miguel, Azores. Life and Marine Sciences, v.24, p.33-40, 2007.

NAVA, D.E.; MELO, M.; NUNES, A.M.; GARCIA, M.S.; BOTTON, M. Mosca em surto. Cultivar Hortaliças e Frutas, v.8, p.26-29, 2008.

OVRUSKI, S.M.; SCHLISERMAN, P.; VAN NIEUWENHOVE, G.A.; BEZDJIAN, L.P.; NÚÑEZ-CAMPERO, S.; ALBORNOZ-MEDINA, P. Occurrence of Ceratitis capitata and Anastrepha fraterculus (Diptera: Tephritidae) on cultivated, exotic fruit species in the highland valleys of Tucuman in Northwest Argentina. Florida Entomologist, v.93, p.277-282, 2010.

PAPACHRISTOS, D.P.; PAPADOPOULOS, N.T.; NANOS, G.D. Survival and development of immature stages of the Mediterranean fruit fly (Diptera: Tephritidae) in citrus fruit. Journal of Economic Entomology, v.101, p.866-872, 2008.

PAPADOPOULOS, N.T.; KATSOYANNOS, B.I.; CAREY, J.R. Demographic parameters of the Mediterranean fruit fly (Diptera: Tephritidae) reared in apples. Annals of the Entomological Society of America, v.95, p.564-569, 2002. 
PLÁCIDO-SILVA, M. do C.; ZUCOLOTO, F.S.; JOACHIM-BRAVO, I.S. Influence of protein on feeding behavior of Ceratitis capitata Wiedemann (Diptera: Tephritidae): comparison between immature males and females. Neotropical Entomology, v.34, p.539-545, 2005.

RAGA, A.; MACHADO, R.A.; SOUZA-FILHO, M.F. de; SATO, M.E.; SILOTO, R.C. Tephritoidea (Diptera) species from Myrtaceae fruits in the state of São Paulo, Brazil. Entomotropica, v.20, p.11-14, 2005.

SALLES, L.A.B. Metodologia de criação de Anastrepha fraterculus (Wiedemann, 1830) (Diptera: Tephritidae) em dieta artificial em laboratório. Anais da Sociedade Entomológica do Brasil, v.21, p.479-486, 1992.

SAS INSTITUTE. Getting started with the SAS learning edition. Cary: SAS Institure, 2002. 200p.

SCOZ, P.L.; BOTTON, M.; GARCIA, M.S. Controle químico de Anastrepha fraterculus (Wied.) (Diptera: Tephritidae) em laboratório. Ciência Rural, v.34, p.1689-1694, 2004.

SILVEIRA-NETO, S.; NAKANO, O.; BARBIN, D.; VILLA-NOVA, N.A. Manual de ecologia dos insetos. Piracicaba: Agronômica Ceres, 1976. 419p.

SOUZA-FILHO, M.F. de; RAGA, A.; AZEVEDO-FILHO, J.A.; STRIKIS, P.C.; GUIMARÃES, J.A.; ZUCCHI, R.A. Diversity and seasonality of fruit flies (Diptera: Tephritidae and Lonchaeidae) and their parasitoids (Hymenoptera: Braconidae and Figitidae) in orchards of guava, loquat and peach. Brazilian Journal of Biology, v.69, p.31-40, 2009.
SOUZA FILHO, M.F. de; RAGA, A.; ZUCCHI, R.A. Moscas-das-frutas no estado de São Paulo: ocorrência e dano. Laranja, v.24, p.45-69, 2003.

THOMAS, M.C.; HEPPNER, J.B.; WOODRUFF, R.E.; WEEMS, H.V.; STECK, G.J.; FASULO, T.R. Mediterranean fruit fly, Ceratitis capitata (Wiedemann) (Insecta: Diptera: Tephritidae), 2010. Available at: <http://edis.ifas.ufl.edu/pdffiles/IN/IN37100. pdf $>$. Accessed on: 02 Mar. 2011.

VALDERRAMA, P.; MARANGONI, F.; CLEMENTE, E. Efeito do tratamento térmico sobre a atividade de peroxidase (POD) e polifenoloxidase (PPO) em maçã (Mallus comunis). Ciência e Tecnologia de Alimentos, v.21, p.321-325, 2001.

ZART, M.; FERNANDES, O.A.; BOTTON, M. Biology and fertility life table of the South American fruit fly Anastrepha fraterculus on grape. Bulletin of Insectology, v.63, p.237-242, 2010.

ZEKI, C.; ER, H.; ÖZDEM, A.; BOZKURT, V. Distribution and infestation of Mediterranean fruit fly (Ceratitis capitata Wied.) (Diptera: Tephritidae) on pome and stone fruits in Isparta and Burdur provinces (Turkey). Munis Entomology and Zoology, v.3, p.231-238, 2008.

ZUCCHI, R.A. Mosca-do-mediterrâneo, Ceratitis capitata (Diptera: Tephritidae). In: VILELA, E.F.; ZUCCHI, R.A.; CANTOR, F. (Ed.). Histórico e impacto das pragas introduzidas no Brasil. Ribeirão Preto: Holos, 2001. p.15-22.

ZUCOLOTO, F.S. Acceptability of different Brazilian fruits to Ceratitis capitata (Diptera: Tephritidae) and fly performance on each species. Brazilian Journal of Medical and Biological Research, v.26, p.291-298, 1993.

Recebido em 11 de abril de 2011 e aprovado em 28 de julho de 2011 